\title{
MOTION OF A COMPRESSIBLE PLATE DRIVEN BY SHOCK OR DETONATION*
}

\author{
BY \\ W. C. RIVARD AND RAY ENGELKE \\ Los Alamos Scientific Laboratory
}

\begin{abstract}
The method of characteristics is used to obtain solutions for the timedependent motion of a compressible plate driven by a one-dimensional shock or detonation followed by a constant state. The plate can either move in contact with or separate from the driver, depending on the values of the initial parameters. The motion of the surfaces is described by a system of linear, first-order, ordinary differential equations in one independent variable. The interior waves and interactions are determined from the surface motions. The equation of state of the plate has the form described by Murnaghan, specialized to produce straight characteristics in the $x, t$ plane. The equation of state for the driver is arbitrary. Closed-form solutions are obtained for the surfaces during their first reverberations for particular driver equations of state. Numerical examples are given which closely represent real systems. The results can be used as standards for finite-difference calculations.
\end{abstract}

1. Introduction. The one-dimensional, time-dependent motion of a compressible plate driven by a step shock or step detonation, i.e. one without a following Taylor wave, is studied. The driver and plate are treated as inviscid fluids so that the Euler equations govern the motion. Both the overmatch (reflected shock) and the undermatch (reflected rarefaction) cases are treated. In either case the plate can separate from the driver for certain values of the initial parameters. The equation of state for the plate has the form described by Murnaghan [1], specialized to produce straight characteristics. The equation of state for the driver is arbitrary, except for satisfaction of the Bethe-Weyl conditions. One set of characteristics in the driver is straight since the flow is composed of simple waves. Solution of the Euler equations under these conditions is reduced to solution of a system of ordinary differential equations which are first-order and linear. In certain cases, closed-form solutions and solutions valid for all time can be obtained.

The solutions display the nonlinear wave interactions which are the mechanism for the acceleration of the interface and the free surface. They also furnish a convenient way to study the effects of the initial parameters. Heretofore, solutions had to be obtained by numerical calculation over a two-dimensional, space-time net. The solutions obtained here can serve as a standard for comparing calculational methods.

* Received October 2, 1972; revised version received March 23, 1973. Work performed under the auspices of the U. S. Atomic Energy Commission. The authors thank Wildon Fickett and W. C. Davis for their stimulating discussions and Harold Langley for his careful preparation of the illustrations. 
Similar boundary value problems have been studied by others. Pack [2] considered an elastic plate driven by a step shock and obtained a closed solution for the plate motion using Hooke's law as the equation of state. Aziz et al. [3] treated an incompressible plate driven by explosion. They obtained a closed solution for the plate motion in the weak shock approximation using a polytropic equation of state with $\gamma=3$ for the detonation products. Kivity and Kogan [4] considered a compressible plate driven by a flying plate of the same material. They obtained a closed solution for the motion over a finite time for certain plate thickness ratios. A Murnaghan equation of state with $\gamma=3$ was used.

The motion of a compressible half-space has also been studied. Ablow [5] treated both the overmatch and the undermatch cases for an arbitrary plane wave in the driver. $\mathrm{He}$ obtained a system of ordinary integro-differential equations for the motion of the interface and the transmitted and reflected waves. The solution holds in the weak shock approximation for Murnaghan equations of state. Fickett [6] treated the undermatch case with a plane, unsupported, Chapman-Jouguet detonation in the driver. He obtained ordinary differential equations for the motion of the interface, transmitted shock, and particle paths. A polytropic equation of state with $\gamma=3$ was used for the detonation products and the half-space was described by an equation of state due to Walsh (see [6]). Kivity and Kogan [7] considered a flying plate driver and obtained a closed solution for the interface motion over a finite time using Murnaghan equations of state with $\gamma=3$.

The equations of motion and the plate equation of state are presented and discussed in Sec. 2. Possible plate motions are discussed in Sec. 3. Secs. 4 and 5 treat motion without separation and with separation respectively. Numerical examples are given in Sec. 6 .

2. Equations of motion, initial and boundary conditions. The equations of motion for the driver and plate are the one-dimensional, time-dependent Euler equations. In characteristic form these are

$$
d x / d t=u \pm c, \quad d p / d u=\mp c / v,
$$

where $x, t, u, c, p$, and $v$ are the position, time, particle velocity, sound speed, pressure, and specific volume. The upper and lower signs correspond to forward and backward characteristics.

The driver equation of state is arbitrary, whereas a special form of the Murnaghan equation of state is used for the plate. The general Murnaghan equation of state is

$$
e(p, v)=(p+\gamma b) v /(\gamma-1)+\text { const. }
$$

For the plate we specialize Eq. (2.3) by setting $\gamma=3$ to obtain

$$
e(p, v)=(p+3 b) v / 2-3 b v_{1} / 2,
$$

where the constant is chosen so that $e\left(0, v_{1}\right)=0$. In Eq. (2.4), $e$ is the specific internal energy, $b$ is a constant related to the initial sound speed, and subscript 1 refers to the initial state. This specialization produces straight characteristics in the plate. This is critical to reducing the problem to ordinary differential equations in one variable. With Eq. (2.4), Eqs. (2.1) and (2.2) become

$$
\begin{gathered}
\left(x-x_{k}\right) /\left(t-t_{k}\right)=u \pm c=u_{k} \pm c_{k}, \\
p_{c}(u ; \mathbf{k}, \mp)=\left(p_{k}+b\right)\left[1 \mp\left(u-u_{k}\right) / c_{k}\right]^{3}-b,
\end{gathered}
$$


where $\mathbf{k}$ is a reference state and $p_{c}$ is the pressure on the characteristic curve through state $\mathbf{k}$. Both sets of characteristics are straight lines in the $x, t$ plane. Eq. (2.4) is easily shown to be the most general form that yields this result for isentropic flow. The following relations derived for Eq. (2.4) are useful later:

a) the equation of an isentrope

$$
p(v, \sigma)=Z(\sigma) v^{-3}-b,
$$

where $Z$ is a function of the entropy, $\sigma$,

b) the sound speed relation

$$
c(p, v)=[3(p+b) v]^{1 / 2},
$$

c) the shock Hugoniot relations

$$
\begin{aligned}
p_{H}(u ; \mathbf{k}, \pm) & =p_{k}+\left(u-u_{k}\right)^{2} / v_{k} \pm\left(u-u_{k}\right)\left[\left(u-u_{k}\right)^{2}+c_{k}^{2}\right]^{1 / 2} / v_{k}, \\
p_{H}(v ; \mathbf{k}) & =\left[\left(p_{k}+3 b\right)\left(v_{k}-v\right)+p_{k} v_{k}\right] /\left(2 v-v_{k}\right), \\
W(u ; \mathbf{k}, \pm) & =u \pm\left[\left(u-u_{k}\right)^{2}+c_{k}^{2}\right]^{1 / 2}
\end{aligned}
$$

where $p_{H}$ is the pressure on the Hugoniot curve, $W$ is the shock velocity through state $\mathbf{k}$, and the upper and lower signs correspond to forward and backward shocks.

A constant-velocity piston at $x=-\infty$ initiates motion in the driver from rest and zero pressure. Time is measured relative to the arrival of the shock or detonation at $x=0$. The interface is initially at $x_{0}$ and the free surface, on which the pressure is zero, is initially at $x_{1}$.

3. Shock/interface interaction and types of motion. The state behind the step shock in the driver is determined when an equation of state, shock velocity, and initial specific volume are specified. For high explosives, similar specification determines either a Chapman-Jouguet, weak, or strong detonation [8]. The initial pressure and particle velocity are zero for both the driver and plate. The shock or detonation interaction at the interface transmits a shock into the plate and reflects a shock, rarefaction, or no wave into the driver accordingly as

$$
\begin{aligned}
& >0, & & \text { reflected shock, } \\
u_{s}{ }^{2} / v_{1} p_{s}-\left(2+3 b / p_{s}\right)^{-1} & =0, & & \text { no reflected wave } \\
& <0, & & \text { reflected rarefaction. }
\end{aligned}
$$

In Eq. (3.1) the subscript $s$ refers to the state behind the initial driver shock or, in the case of a high explosive, the detonation state.

The plate can move in contact with the driver or may separate from it. Both types of motion can occur for any case described by Eq. (3.1). Figs. 1 and 2 show plate motions without separation and with separation. In Fig. 1 a shock is reflected and in Fig. 2 a rarefaction. Separation occurs if the zero-pressure particle velocity for the plate is greater than the same quantity for the driver. This condition is expressed in Appendix A for driver materials described by the general Murnaghan equation of state, Eq. (2.3). Fig. 3 shows the ratio, $\zeta$, of the shock impedance of the plate to that of the driver as a function of the driver pressure $\phi=p_{s} / b$ for an inert driver. The region below a constant $\gamma$ curve corresponds to separation. The sound speed parameter, $b$, is the same for both 


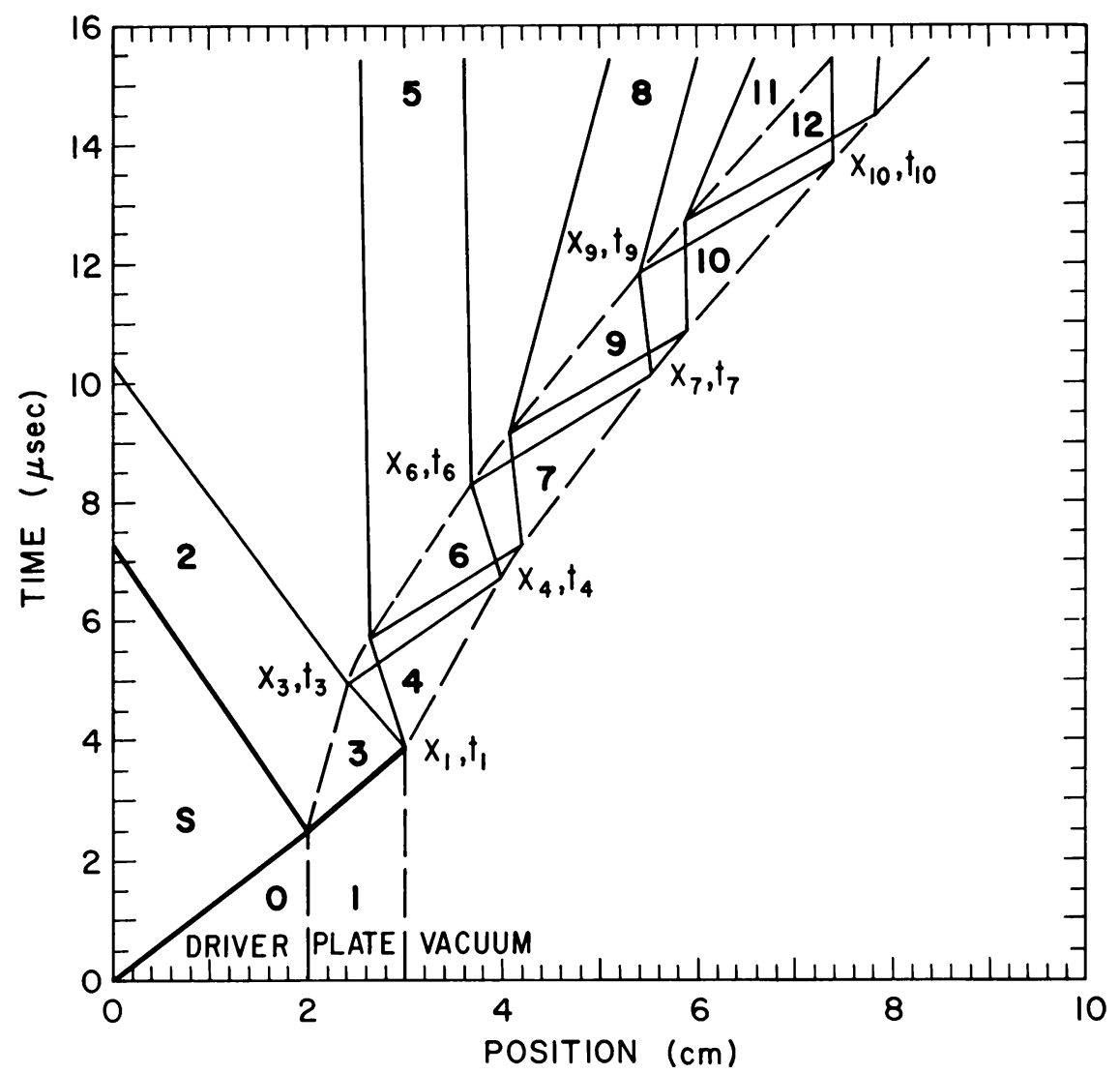

Fig. 1. Plate motion without separation in the overmatch case. The figure corresponds to solution of numerical example 1 in Sec. 6, i.e. a $1.0-\mathrm{cm}$ thick aluminum plate driven by the high explosive Composition B.

the materials. The curves represent solutions of Eqs. (A10), (A13) with $S=0$, and (A14). The region $\zeta>1$ corresponds to an overmatch and $\zeta<1$ to an undermatch.

4. Motion without separation. Fig. 1 shows the simplest wave structure for motion without separation. This structure is common in practical problems. The single forward and backward waves in the plate may, however, become multiple waves for certain initial parameters. By multiple waves we mean, for example, a forward wave composed of a rarefaction and a compression. The differential equations derived below are discussed relative to Fig. 1, but are valid for any wave structure.

The plate motion for an overmatch is considered first. The constant states $\mathbf{2}$ and $\mathbf{3}$, shown in Fig. 1, are determined from the condition that the pressure and particle velocity match across the interface (see Fig. 4). The resulting equation,

$$
p_{H}{ }^{D}\left(u_{2}=u_{3} ; \mathbf{s},-\right)-u_{3}^{2} / v_{1}-u_{3}\left(u_{3}{ }^{2}+c_{1}^{2}\right)^{1 / 2} / v_{1}=0,
$$

is solved for the common particle velocity $u_{3}$, where $p_{2}=p_{H}{ }^{D}\left(u_{2}=u_{3} ; \mathbf{s},-\right)$ and $p_{3}$ is obtained from Eq. (2.9). The superscript $D$ refers to the driver, so that $p_{H}{ }^{D}$ is the Hugoniot pressure in the driver. States 2 and 3 can now be completely determined. In 


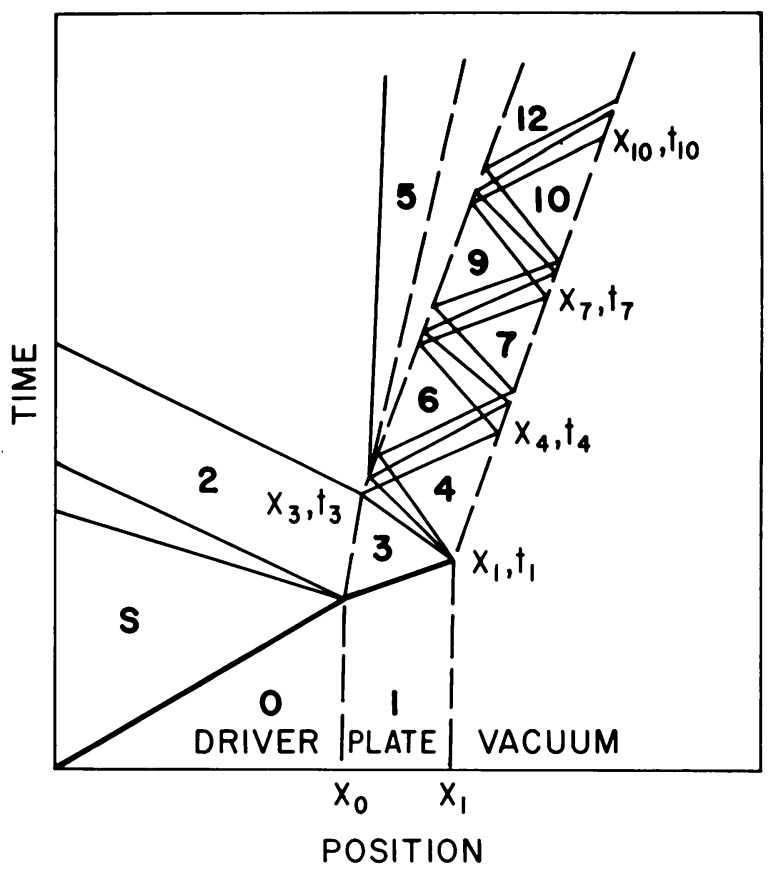

Fig. 2. Sketch of plate motion with separation in the undermatch case.

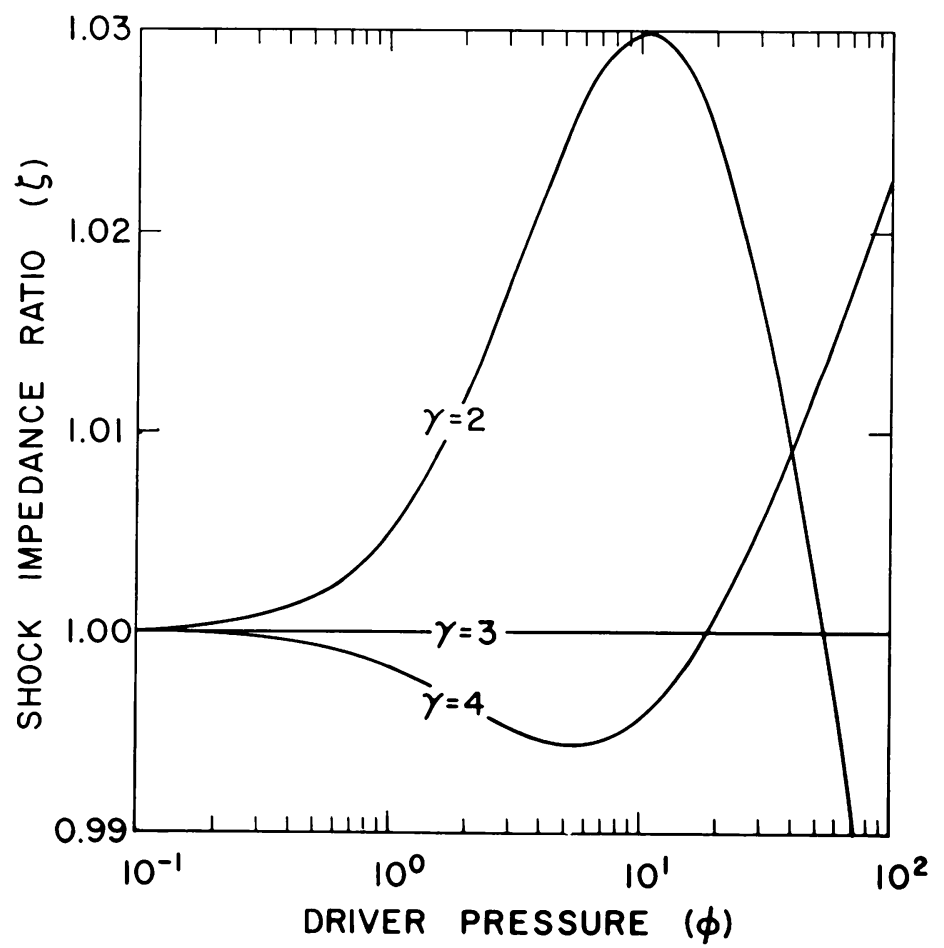

Fig. 3. Shock impedance ratio (plate/driver), $\zeta$, vs. driver pressure, $\phi$, for separation with an inert driver. The curves are solutions of Eqs. (A10), (A13) with $S=0$, and (A14) with $a=b$. Points below a constant- $\gamma$ curve correspond to separation. 
particular, for state 3, $p_{3}, v_{3}, W, c_{3}$ and $Z_{3}$ are determined by Eqs. (2.9)-(2.11), (2.8), and (2.7).

The motion of the plate is isentropic after the initial shock provided none of the compression waves steepens into a shock. Shock formation is checked by calculating the characteristic envelope for each compression wave as described in Appendix B. The motion of the driver is isentropic after the reflected shock until the lead characteristic of the first backward-facing rarefaction overtakes the shock. The forward characteristic emanating from the overtake point provides an upper bound in the $(x, t)$ plane for the driver and plate solutions, provided there is no shock formation. In practical problems this time is long compared to the shock transit-time for the plate, since the reflected shock is generally weak (see Fig. 1). Also, shock formation is uncommon in practical problems, as discussed in Appendix B.

The regions numbered in Fig. 1 are constant states as a result of the step shock or detonation in the driver. The states adjacent to the free surface have $p_{3 n+1}=0$ and

$$
u_{3 n+1}=u_{3 n}+c_{3 n}-c_{3 n+1}, \quad n \geq 1
$$

from Eq. (2.5). Eqs. (2.7) and (2.8) determine $v_{3 n+1}\left(=v_{4}\right)$ and $c_{3 n+1}\left(=c_{4}\right)$. The states adjacent to the interface are determined from the pressure and particle velocity match across the interface. The pressure in the driver $p_{3 n-1}=p_{c}^{D}\left(u_{3 n-1} ; 2,-\right)$, on the forward characteristic through state 2 is equated to the pressure in the plate on the backward characteristic through state $3 \mathrm{n}-2$ given by Eq. (2.6). The resulting equation,

$$
p_{c}{ }^{D}\left(u_{3 n-1}=u_{3 n} ; 2,-\right)-b\left[1+\left(u_{3 n}-u_{3 n-2}\right) / c_{3 n-2}\right]^{3}+b=0,
$$

is solved for the common particle velocity $u_{3 n}$ where $n \geq 2$. States $3 \mathbf{n}$ (plate) and $3 \mathbf{n}-\mathbf{1}$ (driver) are determined from the particle velocity. In particular, for state $3 \mathbf{n}, p_{3 n}, v_{3 n}$, and $c_{3 n}$ are determined by Eqs. (2.6)-(2.8).

In the pressure-particle velocity plane as shown in Fig. 4, the constant states map to points. The rarefaction and compression waves adjacent to the constant states are simple waves and map to forward and backward characteristics respectively $\left(C_{ \pm}\right.$for the plate and $C_{+}{ }^{D}$ for the driver). The forward Hugoniot curve for the plate $\left(H_{+}\right)$through state 1 and the backward Hugoniot curve for the driver $\left(H_{-}{ }^{D}\right)$ through state $\mathbf{S}$ are also shown.

The motions of the interface and the free surface are described by linear, first-order, ordinary differential equations for each rarefaction or compression wave interaction. The interaction beginning at time $t_{3}$ in Fig. 1 is considered the first $(n=1)$ interaction at the interface. Position, time, velocity, and pressure on the interface are denoted by $X_{3 n}, T_{3 n}, U_{3 n}$, and $P_{3 n}$ for the $n$th interaction and are continuous across the interface. Sound speed and specific volume are discontinuous and denoted by $C_{3 n-1}, V_{3 n-1}$ for the driver and $C_{3 n}, V_{3 n}$ for the plate. Similarly, the interaction beginning at time $t_{4}$ is considered the first $(n=1)$ interaction at the free surface. The solution on the free surface for the $n$th interaction is denoted by $X_{3 n+1}, T_{3 n+1}, U_{3 n+1}, P_{3 n+1}(=0), C_{3 n+1}\left(=c_{4}\right)$, and $V_{3 n+1}\left(=c_{4}{ }^{2} / 3 b\right)$. The independent variable for all interactions is the slope $(\eta)$ of the backward characteristics composing the rarefaction wave centered at $x_{1}, t_{1}$;

$$
\eta=\left(X_{3}-x_{1}\right) /\left(T_{3}-t_{1}\right)=U_{3}(\eta)-C_{3}(\eta),
$$

with range $u_{3}-c_{3}=\eta_{3} \leq \eta \leq \eta_{4}=u_{4}-c_{4}$. 


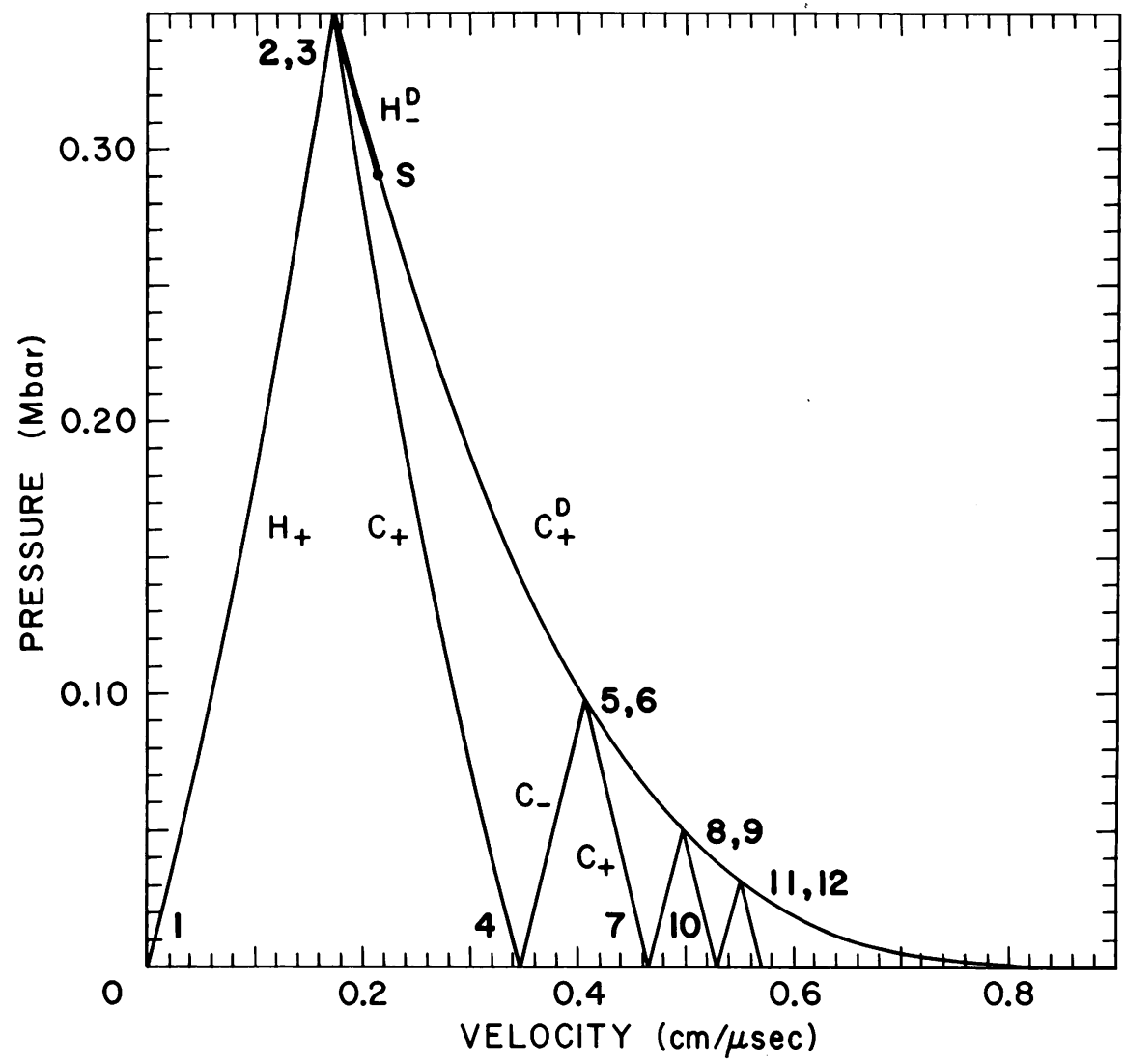

FIg. 4. Pressure-particle velocity plane in the overmatch case. The figure corresponds to solution of numerical example 1 in Sec. 6, i.e. a 1.0-cm-thick aluminum plate driven by the high explosive Composition B.

Time on the interface, $T_{3 n}(\eta)$, during the $n$th interaction is determined by the differential equation

$$
T_{3 n}{ }^{\prime}=\left[c_{4} T_{3 n-2}{ }^{\prime}-2 s^{\prime}\left(T_{3 n}-T_{3 n-2}\right)\right] / C_{3 n},
$$

where prime denotes differentiation with respect to $\eta$. Eq. (4.5) is derived by differentiating Eq. (2.5), $\left(X_{3 n}-X_{3 n-2}\right) /\left(T_{3 n}-T_{3 n-2}\right)=U_{3 n}-C_{3 n}$, and eliminating $X_{m}^{\prime}$ through the particle velocity definition, $X_{m}{ }^{\prime}=U_{m} T_{m}{ }^{\prime}$, where $m=3 n$ and $3 n-2$. The quantity $2 s\left(=C_{3 n}-U_{3 n}\right)$ is the Riemann invariant for the backward characteristic. For the first interaction $(n=1), X_{0}=x_{0}, T_{0}=t_{0}, X_{1}=x_{1}, T_{1}=t_{1}, 2 s=-\eta$ and $2 s^{\prime}=-1$. The sound speed, $C_{3 n}$, is given by

$$
C_{3 n}=U_{3 n}+2 s,
$$

where $U_{3 n}$ is determined from the pressure match at the interface

$$
P_{c}^{D}\left(U_{3 n} ; 2,-\right)-\left(27 Z_{3}\right)^{-1 / 2}\left[U_{3 n}+2 s(\eta)\right]^{3}+b=0,
$$

using Eqs. (2.6), (2.7), and (2.8). The initial condition for Eq. (4.5) is 


$$
T_{3 n}\left(\eta_{3}\right)=t_{3 n-2}+\left\{x_{3 n-2}-X_{3 n-3}\left(\eta_{4}\right)+u_{3 n}\left[T_{3 n-3}\left(\eta_{4}\right)-t_{3 n-2}\right]\right\} / c_{3 n} .
$$

The position, pressure, and specific volume are obtained from Eqs. (2.5), (2.6), and (2.7).

Time on the free surface, $T_{3 n+1}(\eta)$, during the $n$th interaction is determined from the differential equation

$$
T_{3 n+1}^{\prime}=\left[C_{3 n} T_{3 n}^{\prime}-2 r^{\prime}\left(T_{3 n+1}-T_{3 n}\right)\right] / c_{4},
$$

which is derived similarly to Eq. (4.5). The quantity $2 r\left(=U_{3 n}+C_{3 n}\right)$ is the Riemann invariant for the forward characteristic. The derivative $2 r^{\prime}$ is given by $2 r^{\prime}=2\left(U_{3 n}{ }^{\prime}+s^{\prime}\right)$ using Eq. (4.6) where $U_{3 n}{ }^{\prime}$ is obtained by differentiation of Eq. (4.7). The initial condition for Eq. (4.9) is

$$
T_{3 n+1}\left(\eta_{3}\right)=t_{3 n}+\left\{X_{3 n-2}\left(\eta_{4}\right)-x_{3 n}+u_{3 n+1}\left[t_{3 n}-T_{3 n-2}\left(\eta_{4}\right)\right]\right\} / c_{4} .
$$

The velocity is given by $U_{3 n+1}=U_{3 n}+C_{3 n}-c_{4}$ and the position by Eq. (2.5) with reference state $X_{3 n}, T_{3 n}$.

Eqs. (4.8) and (4.10) yield initial conditions for the first $(n=1)$ interactions from the constant-state solutions. Initial conditions for the $n$th interactions can be determined after solution for the $(n-1)$ st interactions.

An interesting special case arises when the driver and plate are described by the same equation of state, Eq. (2.4), but $v_{0} \neq v_{1}$, where $v_{0}$ is the initial specific volume of the driver. In this case Eqs. (4.5) and (4.9) can be integrated in closed form for $n=1$. The solution of Eq. (4.5) is

$$
T_{3}(\eta)=t_{1}+\left(t_{3}-t_{1}\right)\left[c_{3} / C_{3}(\eta)\right]^{(\mu+1)},
$$

where $\mu=\left(Z_{2} / Z_{3}\right)^{1 / 6}$ and $C_{3}(\eta)=\left(u_{2}+c_{2}-\eta\right) /(\mu+1)$. In Eq. (4.9) the quantity $2 r^{\prime}$ is a constant, $(\mu-1) /(\mu+1)$. The solution of Eq. (4.9) is

$$
T_{4}(\theta)=t_{1}+\left(t_{4}-t_{1}\right) e^{\left(\theta-\theta_{3}\right)}-2 \mu\left(t_{3}-t_{1}\right) \theta_{3}{ }^{(\mu+1)} e^{\theta}\left[\Gamma\left(-\mu, \theta_{3}\right)-\Gamma(-\mu, \theta)\right] /(\mu-1),
$$

where $\theta(\eta)=(\mu-1) C_{3}(\eta) / c_{4}, \theta_{3}=\theta\left(\eta_{3}\right)$, and $\Gamma$ is the incomplete gamma function.

The solutions in the interior of the driver and plate are related algebraically to the solutions on the interface and the free surface. The flow in the interior of the driver, following constant state 2, consists of constant states and backward-facing simple rarefactions. At any point $x, t$ in the $n$th rarefaction, we can determine $u$ and $c$ from the interface solution. Since the rarefaction waves are simple waves, the backward characteristics are straight lines and

$$
\begin{aligned}
& u(x, t)=U_{3 n}\left(a_{1}\right), \\
& c(x, t)=C_{3 n}\left(a_{1}\right),
\end{aligned}
$$

where $a_{1}$ is the root of $H_{3 n-1}\left(a_{1} ; x, t,-\right)=0$ and

$$
H_{m}(\eta ; x, t, \pm) \equiv x-X_{m}(\eta)-\left[U_{m}(\eta) \pm C_{m}(\eta)\right]\left[t-T_{m}(\eta)\right]
$$

Note that $X_{3 n-1}=X_{3 n}, T_{3 n-1}=T_{3 n}$, and $U_{3 n-1}=U_{3 n}$, since these quantities are continuous across the interface. The pressure is given by $p(x, t)=p_{c}{ }^{D}[u(x, t) ; 2,-]$ and the specific volume is determined from the $p(v)$ isentrope through state 2 .

The flow in the interior of the plate, following constant state 3 , consists of constant states, simple rarefactions and compressions, and interactions. At any point $x, t$ in the $n$th rarefaction or compression, two equations for $u$ and $c$ can be obtained from Eq. (2.5): 


$$
\begin{gathered}
u(x, t)=\frac{1}{2}\left[U_{3 n}\left(a_{1}\right) \mp C_{3 n}\left(a_{1}\right)+u_{3 n+1} \pm c_{3 n+1}\right] \\
c(x, t)=c_{3 n+1} \pm u_{3 n+1} \mp u(x, t),
\end{gathered}
$$

where $a_{1}$ is the root of $H_{3 n}\left(a_{1} ; x, t, \mp\right)=0$. The pressure is given by Eq. (2.6) as $p(x, t)=$ $p_{c}\left[u(x, t) ; a_{1}, \pm\right]$, where reference state $a_{1}$ means $U_{3 n}\left(a_{1}\right), C_{3 n}\left(a_{1}\right)$, and $P_{3 n}\left(a_{1}\right)$. The specific volume is given by Eq. (2.7). The upper sign is used for the rarefactions and the lower sign for the compressions.

Similarly, for any point $x, t$ in the $n$th interaction region at the interface and at the free surface,

$$
\begin{aligned}
& u(x, t)=\frac{1}{2}\left[U_{3 n}\left(a_{1}\right)+C_{3 n}\left(a_{1}\right)+U_{m}\left(a_{2}\right)-C_{m}\left(a_{2}\right)\right], \\
& c(x, t)=C_{3 n}\left(a_{1}\right)+U_{3 n}\left(a_{1}\right)-u(x, t) .
\end{aligned}
$$

In Eq. (4.18), $a_{1}$ is the root of $H_{3 n}\left(a_{1} ; x, t,+\right)=0, a_{2}$ is the root of $H_{m}\left(a_{2} ; x, t,-\right)=0$, and $m=3 n$ and $3 n+1$ for interaction regions adjacent to the interface and the free surface respectively. The pressure and specific volume are calculated as for the simple waves.

We now consider the plate motion for an undermatch. In this case states $\mathbf{s}$ and 2 are joined by a backward-facing, centered, simple rarefaction. Consequently, state 2 lies on the forward characteristic through state $\mathrm{s}$. The particle velocity, $u_{2}=u_{3}$, is determined from the relation

$$
p_{c}{ }^{D}\left(u_{2}=u_{3} ; \mathbf{s},-\right)-u_{3}{ }^{2} / v_{1}-u_{3}\left(u_{3}{ }^{2}+c_{1}{ }^{2}\right) / v_{1}=0,
$$

in place of Eq. (4.1).

The remaining constant states and the motions of the interface and the free surface are determined by the equations for the overmatch case. The solution can be determined for all time in the absence of shock formation in the plate. In the interior of the centered rarefaction reflected into the driver, the solution is determined by the relation $u(x, t)-$ $c(x, t)=\left(x-x_{0}\right) /\left(t-t_{0}\right)$ and the constancy of the Riemann invariant on the forward characteristic through state 2 .

5. Motion with separation. The plate may separate from the driver for certain values of the initial parameters. This generally occurs during the first interface interaction but may occur during a later interaction. Fig. 3 shows regions in the $\zeta, \phi$ plane where separation takes place. Fig. 2 shows a sketch of the motion in the $x, t$ plane. The double wave structure (e.g. a forward wave composed of a rarefaction and a compression) in the plate is the simplest structure for this type of motion. As in Sec. 4, the differential equations derived below are valid for any wave structure.

In the following we restrict our attention to the case where separation occurs during the first interaction, at time $T_{3}\left(\eta_{c}\right)$, where $\eta_{3} \leq \eta_{c} \leq \eta_{4}$. The pressure at the separation point is $P_{3}\left(\eta_{c}\right)=0$, the velocity is $U_{3}\left(\eta_{c}\right)=u_{5}$, and the rate of separation is $\Delta u=$ $u_{4}-u_{5}=\eta_{4}-\eta_{c}$. A sketch of the pressure-particle velocity plane is shown in Fig. 5 . The motion induces tension (negative pressure) in the plate, the maximum tension being $p=b\left[1-\left(u_{4}-u_{\min }\right) / 2 c_{4}\right]^{3}-b$, where $u_{\min }$ is the minimum velocity of the free surface.

Eqs. (4.5) and (4.9) determine $T_{3}$ and $T_{4}$ for $\eta \leq \eta_{c}$. These equations simplify for $\eta>\eta_{c}$ to 


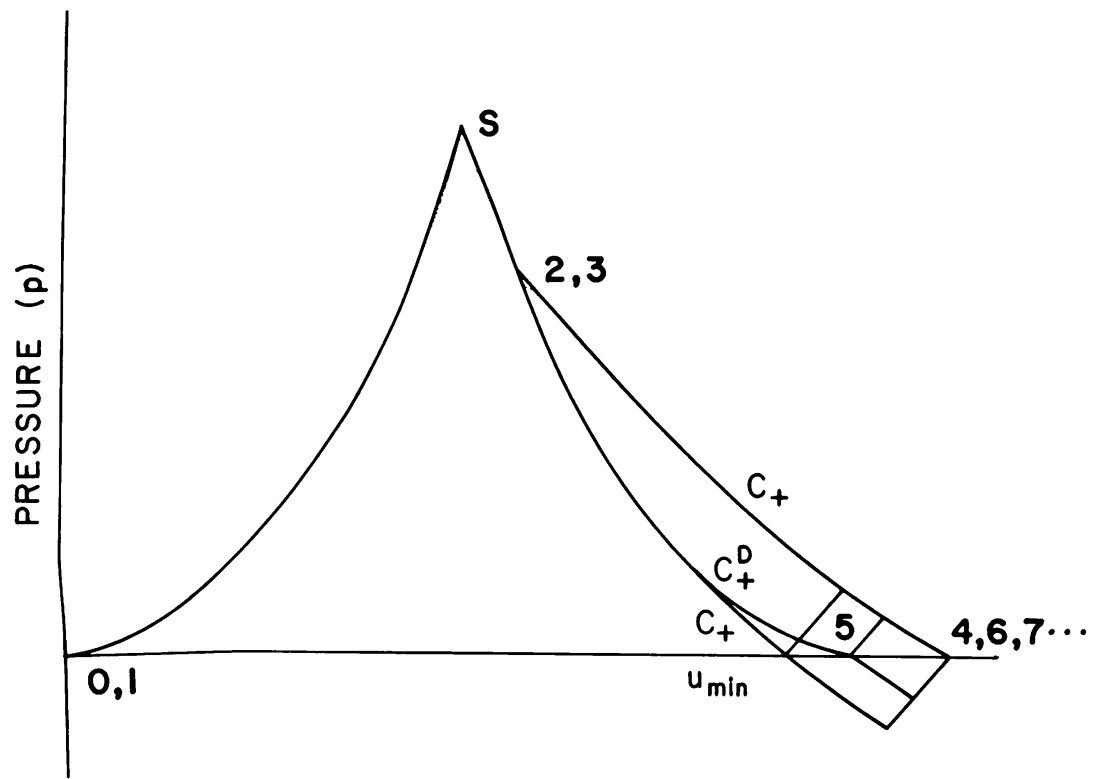

VELOCITY (u)

Fig. 5. Sketch of the pressure-particle velocity plane for motion with separation in the undermatch case. The figure corresponds to numerical example 2 in Sec. 6, i.e. a 1.0-cm-thick rhodium plate driven by tantalum.

$$
\begin{aligned}
T_{3 n}{ }^{\prime} & =T_{3 n-2}{ }^{\prime}+\left(T_{3 n}-T_{3 n-2}\right) / c_{4}, \\
T_{3 n+1}{ }^{\prime} & =T_{3 n}{ }^{\prime}-\left(T_{3 n+1}-T_{3 n}\right) / c_{4},
\end{aligned}
$$

since the interface becomes a zero-pressure boundary. Eqs. (5.1) and (5.2) also hold when $\eta \leq \eta_{c}$ and $n \geq 2$, since separation occurs during the first interaction. These equations can be integrated in closed form for the initial conditions given by Eqs. (4.8) and (4.10). The solutions for time on the back surface are

$$
\begin{array}{ll}
n=1: & T_{3}\left(\eta \leq \eta_{c}\right) \cdots \text { from Eq. (4.5), } \\
& T_{3}\left(\eta>\eta_{c}\right)=t_{1}+\Delta \tau_{1} e^{\psi}, \\
n \geq 2: & T_{3 n}\left(\eta \leq \eta_{c}\right)=T_{4}\left(\eta \leq \eta_{c}\right)+(n-1) \Delta \tau_{1} e^{\omega}+(n-2) \Delta \tau_{2} e^{-\omega}, \\
& T_{3 n}\left(\eta>\eta_{c}\right)=t_{1}+n \Delta \tau_{1} e^{\psi}+(n-1) \Delta \tau_{2} e^{-\psi} .
\end{array}
$$

Similarly, the solutions for time on the free surface are

$$
\begin{array}{ll}
n=1: & T_{4}\left(\eta \leq \eta_{c}\right) \cdots \text { from Eq. (4.9), } \\
& T_{4}\left(\eta>\eta_{c}\right)=t_{1}+\Delta \tau_{1} e^{\psi}+\Delta \tau_{2} e^{-\psi} \\
n \geq 2: & T_{3 n+1}\left(\eta \leq \eta_{c}\right)=T_{4}\left(\eta \leq \eta_{c}\right)+(n-1)\left(\Delta \tau_{1} e^{\omega}+\Delta \tau_{2} e^{-\omega}\right), \\
& T_{3 n+1}\left(\eta>\eta_{c}\right)=t_{1}+n\left(\Delta \tau_{1} e^{\psi}+\Delta \tau_{2} e^{-\psi}\right),
\end{array}
$$

where $\Delta \tau_{1}=\left[T_{3}\left(\eta_{c}\right)-t_{1}\right] \exp \left(\Delta u / c_{4}\right), \Delta \tau_{2}=\left[T_{4}\left(\eta_{c}\right)-T_{3}\left(\eta_{c}\right)\right] \exp \left(-\Delta u / c_{4}\right), \psi=$ $\left(\eta-\eta_{4}\right) / c_{4}, \omega=\left[U_{4}(\eta)-u_{4}\right] / c_{4}$, and $U_{4}(\eta)$ is a result of solution for $T_{4}$. The additional flow variables and the interior solutions are determined as described in Sec. 4. 
Shock formation in both the forward and backward compression waves is checked as described in Appendix B. Shock formation is more common here than for motion without separation. In fact, a class of motions is described in Appendix B for which shock formation will always occur.

6. Numerical examples. Two numerical examples are given to illustrate motion without and with separation. In each example the driver is described by the general Murnaghan equation of state

$$
e(p, v)=\left[(p+\gamma a) v-\gamma a v_{0}\right] /(\gamma-1),
$$

where $e\left(0, v_{0}\right)=0$ and $a$ is a constant related to the initial sound speed. The characteristic and Hugoniot equations for the driver indicated in Eqs. (4.1), (4.3), and (4.7) are

$$
\begin{aligned}
& p_{c}{ }^{D}(u ; \mathbf{k}, \mp)=\left(p_{k}+a\right)\left[1 \mp(\gamma-1)\left(u-u_{k}\right) / 2 c_{k}\right]^{2 \gamma /(\gamma-1)}-a, \\
& p_{H}{ }^{D}(u ; \mathbf{k}, \pm)=p_{k}+(\gamma+1)\left(u-u_{k}\right)^{2} / 4 v_{k} \pm\left(u-u_{k}\right)\left\{\left[(\gamma+1)\left(u-u_{k}\right) / 2\right]^{2}\right. \\
&\left.+4 v_{k} \gamma\left(p_{k}+a\right)\right\}^{1 / 2} / 2 v_{k} .
\end{aligned}
$$

For the first example we consider a 1.0-cm-thick aluminum plate driven by the high explosive Composition B. The initial sound speed and density of the aluminum are $0.5328 \mathrm{~cm} / \mu \mathrm{sec}$ and $2.785 \mathrm{~g} / \mathrm{cm}^{3}$. The constant $b$ in Eq. (2.4) is determined from these values and Eq. (2.8). Eqs. (2.6) and (2.9) with this value of $b$ reproduce experimental isentrope and Hugoniot data $[9,10]$ to within about $4 \%$ over the pressure range of interest. The detonation products are described by Eqs. (6.1)-(6.3) with $\gamma=2.769$ and $a=0$ after Deal [11]. The detonation velocity is $0.7991 \mathrm{~cm} / \mu \mathrm{sec}$ and the initial density is $1.714 \mathrm{~g} / \mathrm{cm}^{3}$.

The solutions in the $x, t$ and $p, u$ planes are shown in Figs. 1 and 4 . There is no shock formation from the compression waves in the plate. Hence, the solution is valid below the forward characteristic emanating from the point $(x=-16.30, t=46.40)$, where the lead characteristic of the first backward-facing rarefaction in the driver overtakes the reflected shock. The initial slope of the forward characteristic is $u_{2}+c_{2}=0.7948$. The history of the free-surface velocity is shown in Fig. 6. Curve B is a magnification of the early portion of curve A. The free-surface accelerations are nearly constant in this example. The dashed line is the steady solution, $u_{\infty}=u_{2}+1.131 c_{2}$, assuming the backward rarefactions in the driver are simple waves for all time.

A fourth-order Runge-Kutta method was used to integrate the system of differential equations, Eqs. (4.5) and (4.9). Initial conditions for the $n=1$ equations were calculated from the constant-state solutions using Eqs. (4.8) and (4.10). The $n=1$ equations were then integrated to obtain the solutions for the first interactions and the results were used to calculate initial conditions for the $n=2$ equations. The $n=1$ equations were integrated again together with the $n=2$ equations to obtain solutions for the second interactions and the results were used to calculate initial conditions for the $n=3$ equations. This procedure was continued for the desired number of interactions. The reintegration of the differential equations was performed because the interval determining Runge-Kutta routine required solution values for the earlier interactions off the previous $\eta$ mesh.

The second example illustrates motion with separation. We consider a 1.0-cm-thick rhodium plate driven by tantalum. The plate is described by $\rho_{1}=11.708 \mathrm{~g} / \mathrm{cm}^{3}$ and 


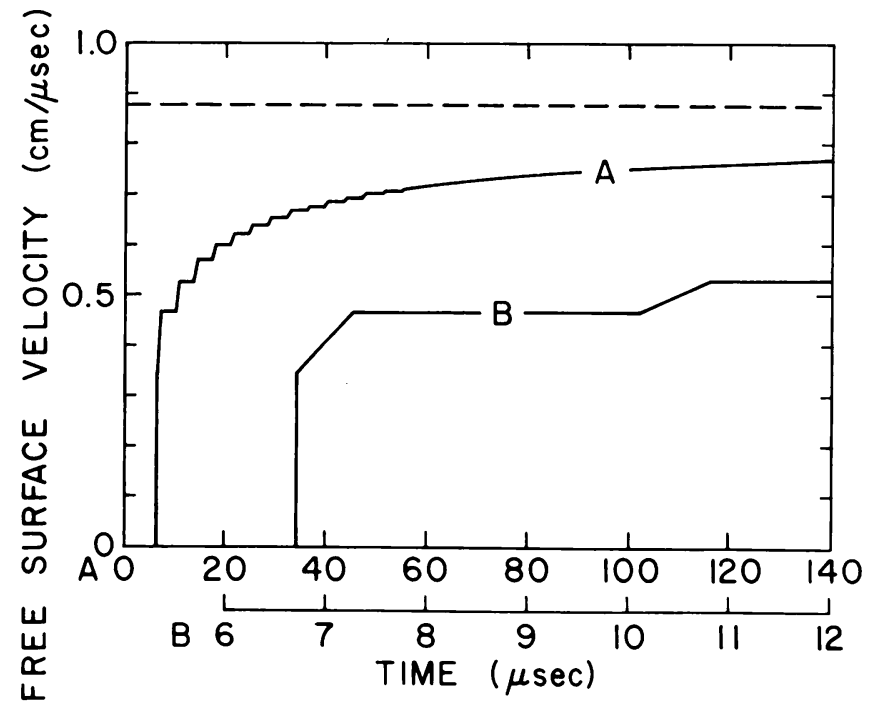

FIG. 6. Free-surface velocity history for numerical example 1 in Sec. 6, i.e. a 1.0-cm-thick aluminum plate driven by the high explosive Composition B.

$c_{1}=0.4987 \mathrm{~cm} / \mu \mathrm{sec}$. The driver has $\rho_{0}=16.654 \mathrm{~g} / \mathrm{cm}^{3}, c_{0}=0.3414 \mathrm{~cm} / \mu \mathrm{sec}$, and $\gamma=2$. The constant $a$ in Eq. (6.1) is determined from $a=c_{0}{ }^{2} \rho_{0} / \gamma$. These parameters describe experimental Hugoniot data [10] to within about 5\% for tantalum and $10 \%$ for rhodium. The initial shock velocity in the tantalum is $1.901 \mathrm{~cm} / \mu \mathrm{sec}$, and the interface and free surface are at $x_{0}=2.0 \mathrm{~cm}$ and $x_{1}=3.0 \mathrm{~cm}$.

Separation occurs during the first interaction at $\eta_{c}=2.138 \mathrm{~cm} / \mu \mathrm{sec}$. The maximum tension in the plate is about $0.1756 \mathrm{Mbar}$ and the rate of separation is $\Delta u=0.0465$ $\mathrm{cm} / \mu$ sec. The solutions for the first interactions at the interface and free surface are given in Table 1. Solutions for the later interactions can be obtained from Table 1 and Eqs. (5.3) and (5.4). Sketches of the pressure-particle velocity plane and the free-surface velocity history are shown in Figs. 5 and 7.

Appendix A. The condition for separation of the plate from the driver during the first interface interaction is $u_{4}\left(p=0, Z_{3}\right)>u_{5}\left(p=0, Z_{2}\right)$. For driver materials described

TABLE 1.

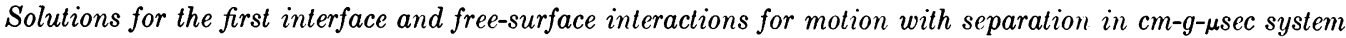

\begin{tabular}{ccccccccc}
\hline \multicolumn{1}{c}{$\eta$} & $X_{3}$ & $T_{3}$ & $U_{3}$ & $C_{3}$ & $P_{3}$ & $V_{3}$ & $X_{4}$ & $T_{4}$ \\
\hline-1.0218 & 2.7662 & 1.6676 & 1.2450 & 2.2667 & 37.695 & 0.0443 & 7.4508 & 3.0016 \\
-0.6655 & 2.8206 & 1.7085 & 1.4102 & 2.0757 & 28.721 & 0.0484 & 7.9835 & 3.1895 \\
-0.3093 & 2.9002 & 1.7616 & 1.5774 & 1.8867 & 21.327 & 0.0532 & 8.5718 & 3.3988 \\
0.0469 & 3.0185 & 1.8326 & 1.7469 & 1.7000 & 15.339 & 0.0591 & 9.2308 & 3.6349 \\
0.4032 & 3.1982 & 1.9304 & 1.9188 & 1.5156 & 10.587 & 0.0662 & 9.9845 & 3.9064 \\
0.7594 & 3.4795 & 2.0703 & 2.9034 & 1.3340 & 6.9098 & 0.0753 & 10.872 & 4.2273 \\
1.1156 & 3.9384 & 2.2800 & 2.2710 & 1.1553 & 4.1489 & 0.0869 & 11.962 & 4.6219 \\
1.4719 & 4.7299 & 2.6142 & 2.4520 & 0.9801 & 2.1550 & 0.1024 & 13.379 & 5.1342 \\
2.1378 & 8.7153 & 4.1123 & 2.8015 & 0.6637 & 0. & 0.1513 & 17.971 & 6.7832 \\
2.1843 & 9.2638 & 4.3065 & 2.8480 & 0.6637 & 0. & 0.1513 & 18.008 & 6.7965 \\
\hline
\end{tabular}




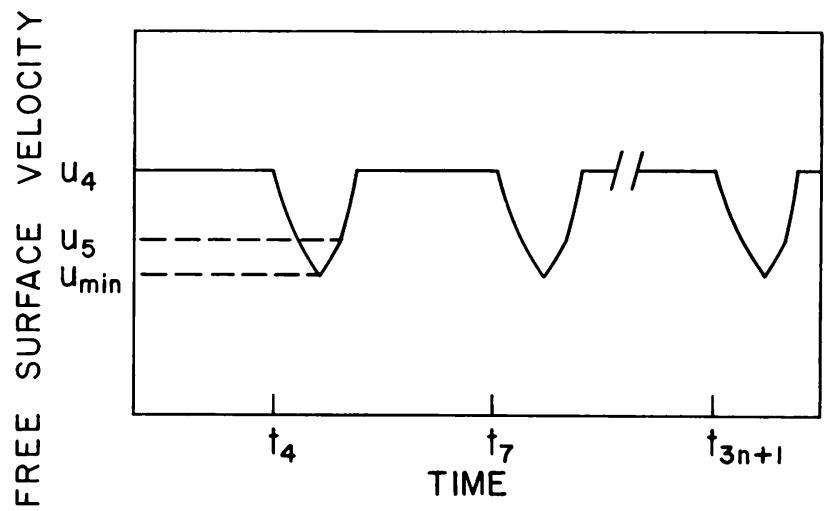

Fig. 7. Sketch of the free-surface velocity history for numerical example 2 in Sec. 6, i.e. a 1.0-cm-thick rhodium plate driven by tantalum.

by Eq. (6.1), this condition can be written as

$$
c_{3}\left(p_{3}, v_{3}\right)-c_{4}\left(0, v_{4}\right)-[2 /(\gamma-1)]\left[c_{2}\left(p_{3}, v_{2}\right)-c_{5}\left(0, v_{5}\right)\right]>0,
$$

since states 2 and 3 are joined to states 5 and 4 by simple waves. Eq. (A1) is rewritten to yield a relation between the initial parameters $\nu=v_{0} / v_{1}$ and $\phi=p_{s} / b$.

The quantity $c_{3}-c_{4}$ is

$$
c_{3}-c_{4}=\left(b v_{0}\right)^{1 / 2}\left[3\left(\phi_{3}+1\right)\left(\phi_{3}+3\right) / \nu\left(2 \phi_{3}+3\right)\right]^{1 / 2}\left[1-\left(\phi_{3}+1\right)^{-1 / 3}\right],
$$

by use of Eqs. (2.6), (2.7) and (2.9), where $\phi_{3}=p_{3} / b$. Similarly, $c_{2}-c_{5}$ is

$$
c_{2}-c_{5}=\left(b v_{0}\right)^{1 / 2}\left[\gamma\left(\phi_{3}+\alpha\right) v_{2} / v_{0}\right]^{1 / 2}\left\{1-\left[\alpha /\left(\phi_{3}+\alpha\right)\right]^{(\gamma-1) / 2 \gamma}\right\},
$$

where $\alpha=a / b$ and the relations

$$
\begin{aligned}
p(v, \sigma) & =Z v^{-\gamma}-a \\
c(p, v) & =[\gamma(p+a) v]^{1 / 2} \\
p_{H}(v ; \mathbf{k}) & =\frac{p_{k}\left[2 v_{k}+(\gamma-1)\left(v_{k}-v\right)\right]+2 \gamma a\left(v_{k}-v\right)}{2 v-(\gamma-1)\left(v_{k}-v\right)}
\end{aligned}
$$

derived for Eq. (6.1) have been used.

The ratio $v_{2} / v_{0}$ in Eq. (A3) is

$v_{2} / v_{0}=\left(v_{s} / v_{0}\right)\left[(\gamma-1) \phi_{3}+(\gamma+1) \phi+2 \gamma \alpha\right] /\left[(\gamma-1) \phi+(\gamma+1) \phi_{3}+2 \gamma \alpha\right]$,

for an overmatch and

$$
v_{2} / v_{0}=\left(v_{s} / v_{0}\right)\left[(\phi+\alpha) /\left(\phi_{3}+\alpha\right)\right]^{1 / \gamma},
$$

for an undermatch. In Eqs. (A7) and (A8),

$$
v_{s} / v_{0}=\Gamma /(\Gamma+1),
$$

where $\Gamma=(\gamma-1) / 2+\gamma \alpha / \phi$ for an inert driver and $\Gamma=\gamma(1+\alpha / \phi)$ for a high explosive driver under Chapman-Jouguet conditions.

Eq. (A1) is now a function of $\nu, \phi$, and $\phi_{3}$. We obtain the desired result in parametric form by expressing $\nu=v_{0} / v_{1}$ as a function of $\phi$ and parameter $\phi_{3}$. Solution of Eq. (2.9) 
for $\nu$ yields

$$
\nu=\phi_{3}{ }^{2} / \lambda^{2}\left(2 \phi_{3}+3\right),
$$

where $\lambda=\left(b v_{0}\right)^{-1 / 2} u_{3}$ and the reference state is 1 . For an overmatch, $\lambda\left(\phi, \phi_{3}\right)$ is given by solution of Eq. (6.3),

$\lambda=\left\{\phi^{1 / 2}-\left(\phi_{3}-\phi\right) \Gamma^{1 / 2}\left[\gamma(\phi+\alpha)+\left(\phi_{3}-\phi\right)(\gamma+1) / 2\right]^{-1 / 2}\right\}(\Gamma+1)^{-1 / 2}$,

using Eq. (A9). For an undermatch, $\lambda\left(\phi, \phi_{3}\right)$ is given by solution of Eq. (6.2),

$\lambda=\left[\phi^{1 / 2}+[2 /(\gamma-1)][\gamma(\phi+\alpha) \Gamma]^{1 / 2}\left\{1-\left[\left(\phi_{3}+\alpha\right) /(\phi+\alpha)\right]^{(\gamma-1) / 2 \gamma}\right\}\right](\Gamma+1)^{-1 / 2}$,

using Eqs. (A4), (A9), and the mechanical shock conditions.

Eq. (A1) can be rewritten using the above results to yield

$$
\begin{aligned}
S\left(\phi ; \phi_{3}\right) \equiv & \left(\lambda / \phi_{3}\right)\left[3\left(\phi_{3}+1\right)\left(\phi_{3}+3\right)\right]^{1 / 2}\left[1-\left(\phi_{3}+1\right)^{-1 / 3}\right] \\
& -[2 /(\gamma-1)]\left[\gamma\left(\phi_{3}+\alpha\right) v_{2} / v_{0}\right]^{1 / 2}\left\{1-\left[\alpha /\left(\phi_{3}+\alpha\right)\right]^{(\gamma-1) / 2 \gamma}\right\}>0,
\end{aligned}
$$

with $\nu\left(\phi ; \phi_{3}\right)$ given by Eq. (A10). The rate of separation is $S=\left(b v_{0}\right)^{-1 / 2}\left(u_{4}-u_{5}\right)$. The impedance ratio plotted in Fig. 3 is

$$
\zeta=W \rho_{1} / W_{D} \rho_{0}=\left[\nu\left(2 \phi_{3}+3\right) / \phi(\Gamma+1)\right]^{1 / 2} .
$$

Appendix B. Shock formation in the plate is discussed in this appendix. The point of shock formation is the earliest time on the envelope formed by the straight characteristics composing a forward or backward compression wave. The characteristics are described by

$$
H_{m}(x, t ; \eta, \pm) \equiv x-X_{m}(\eta)-\left[U_{m}(\eta) \pm C_{m}(\eta)\right]\left[t-T_{m}(\eta)\right]=0,
$$

where $\eta$ is the family parameter and $m=3 n$ and $3 n+1$ for forward and backward waves. Necessary conditions for the existence of an envelope are $H_{m}=0$ and $\partial H_{m} / \partial \eta=0$ which yield

$$
\begin{aligned}
t(\eta) & =T_{m} \pm C_{m} T_{m}{ }^{\prime} /\left(U_{m}{ }^{\prime} \pm C_{m}{ }^{\prime}\right), \\
x(\eta) & =X_{m} \pm C_{m} T_{m}{ }^{\prime}\left(U_{m} \pm C_{m}\right) /\left(U_{m}{ }^{\prime} \pm C_{m}{ }^{\prime}\right) .
\end{aligned}
$$

Eqs. (B2) and (B3) are sufficient to define the envelope, if $\left(\partial H_{m} / \partial x\right)^{2}+\left(\partial H_{m} / \partial t\right)^{2} \neq 0$ and $(\partial x / \partial \eta)^{2}+(\partial t / \partial \eta)^{2} \neq 0$. The first of these conditions is satisfied since $\partial H_{m} / \partial x=1$. The second condition must in general be checked.

Comments on shock formation for motion without separation. Shock formation in practical problems is uncommon, except for large input shock strengths. Fig. 8 shows the locus of shock formation points at the free surface of the plate in the first compression wave. The case illustrated uses Eq. (2.4) for the equation of state of the driver and plate, with $v_{0} \neq v_{1}$. Points lying above the curve correspond to shock formation. For this particular case both envelope sufficiency conditions are satisfied, shock formation occurs on the lead characteristic, and there is no shock formation for an undermatch.

Comments on shock formation for motion with separation. Shock formation in the forward compression wave initiated at the separation point $T_{3}\left(\eta_{c}\right)$ is a common feature for this type of motion. In fact, a shock will always form in the plate if the free-surface 


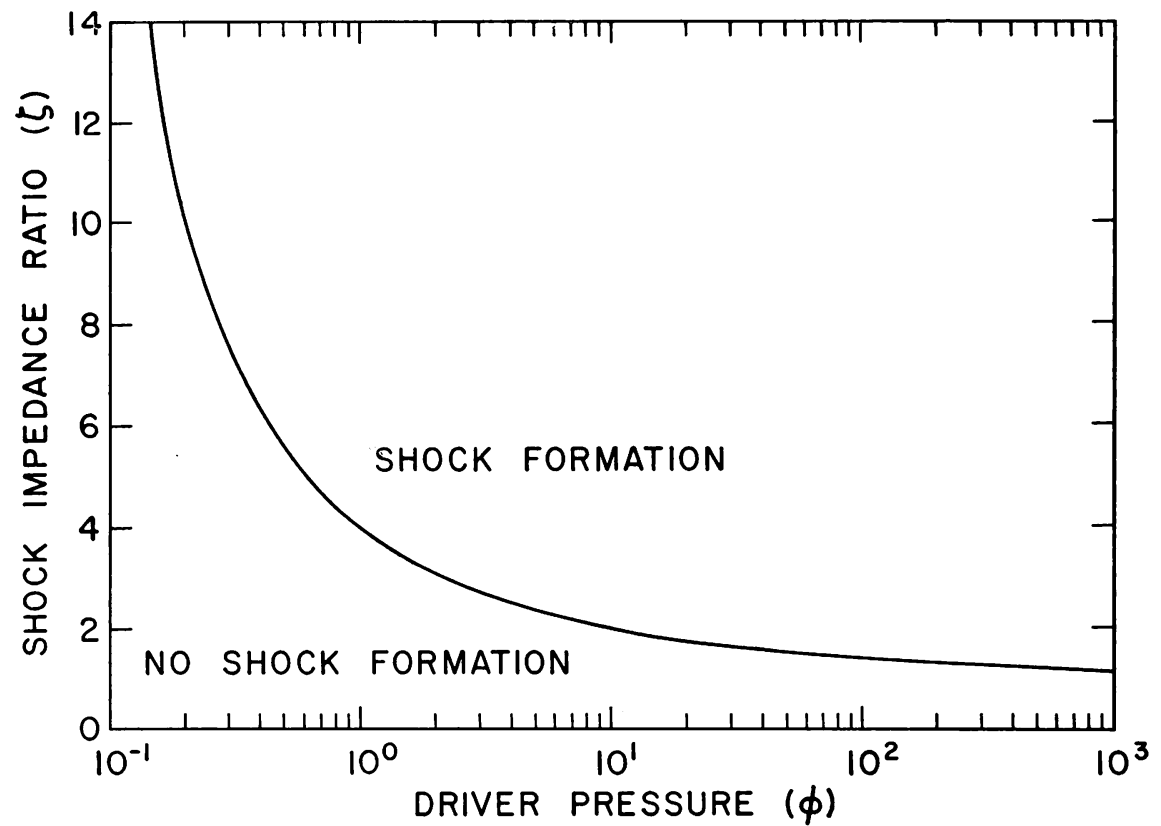

Fig. 8. Shock impedance ratio (plate/driver), $\zeta$, vs. driver pressure, $\phi$, for shock formation at the free surface. The driver and plate have identical equations of state, given by Eq. (2.4), but different initial densities.

velocity is greater than or equal to $u_{5}$ for all $t>t_{1}$. This is easily shown as follows. Eqs. (B2) and (B3) become $t=2 T_{3}-t_{1}$ and $x=X_{3}+2 r\left(T_{3}-t_{1}\right)$ for $\eta \geq \eta_{c}$. Both envelope sufficiency conditions are satisfied and the minimum time on the envelope is $t\left(\eta_{c}\right)$. This time is also the time at which a straight line through $x_{1}, t_{1}$ with slope $u_{5}$ intersects the lead characteristic of the compression wave. Thus, the envelope point $x\left(\eta_{c}\right), t\left(\eta_{c}\right)$ lies inside the plate when the free-surface velocity is greater than or equal to $u_{5}$ for all $t>t_{1}$.

\section{REFERENCES}

[1] F. D. Murnaghan, Finite deformation of an elastic solid, Wiley, New York, 1951, pp. 71-79

[2] D. C. Pack, The reflection and transmission of shock waves, Phil. Mag. 2, 182-195 (1957)

[3] A. K. Aziz, H. Hurwitz, and H. M. Sternberg, Energy transfer to a rigid piston under detonation loading, in Proceedings Third Symposium on Detonation (Princeton Univ., 1960) ONR Symp. Rpt. ACR-52, Vol. 1, pp. 205-225

[4] Y. Kivity and A. Kogan, Analytical solution of the flow field produced by the impact of finite plates, Israel J. Tech. 7, 457-464 (1969)

[5] C. M. Ablow, Wave refraction at an interface, Quart. Appl. Math. 18, 15-29 (1960)

[6] W. Fickett, Motion of an explosive-induced plane shock wave, Quart. Appl. Math., this issue

[7] Y. Kivity and A. Kogan, Interaction at the interface of two colliding plates, Israel J. Tech. 8, 325-328 (1970)

[8] R. Courant and K. O. Friedrichs, Supersonic flow and shock waves, Interscience, New York, 1948

[9] J. M. Walsh, M. H. Rice, R. G. McQueen, and F. L. Yarger, Shock-wave compressions of twentyseven metals: equations of state of metals, Phys. Rev. 108, 196-216 (1957)

[10] Group GMX-6, Los Alamos Scientific Laboratory, Selected Hugoniots, Report LA-4167-MS, 1969

[11] W. E. Deal, Measurement of the reflected shock Hugoniot and isentrope for explosive reaction products, Phys. Fluids 1, 523-527 (1958) 